11

\title{
Анализ магнитокалорического эффекта в кристалле ферромагнитного сплава с памятью формы
}

\author{
(C) Г.А. Малыгин \\ Физико-технический институт им. А.Ф. Иофрфе, \\ Санкт-Петербург, Россия \\ E-mail: malygin.ga@mail.ioffe.ru \\ Поступила в Редакцию 20 декабря 2021 г. \\ В окончательной редакции 20 декабря 2021 г. \\ В окончательной редакции 21 декабря 2021 г.
}

В рамках теории размытых термоупругих мартенситных переходов (РТМП), основанной на термодинамических и кинетических соотношениях, теоретически обсуждается механизм магнитокалорического эффекта в кристалле ферромагнитного сплавов, испытывающего магнитоструктурный (мартенситный) переход. На примере кристалла сплава гейслеровского типа близкого к стехиометрическому составу продемонстрировано, что теория адекватно описывает магнитокалорический эффект в этом сплаве при адиабатическом включении и выключении магнитного поля и учитывает влияние на него магнитной анизотропии кристалла.

Ключевые слова: магнитоструктурные мартенситные переходы, ферромагнитные сплавы гейслеровского типа, изотермическое изменение энтропии, магнитокалорический эффект.

DOI: 10.21883/FTT.2022.05.52339.258

\section{1. Введение}

Сплавы с эффектом памяти формы (ЭПФ) обладают целым комплексом функциональных свойств, стимулирующих их использование в качестве сенсорных и исполнительных элементов в различных устройствах микро- и наноэлектроники, робототехники, медицины и промышленности. Широкому применению сплавов способствует их чувствительность к механическим напряжениям, магнитным и электрическим полям. Внимание исследователей в последнее время привлечено к изучению эффекта адиабатического разогрева и охлаждения кристаллов этих сплавов вблизи критической температуры мартенситного (структурного) в них перехода. Эффект возникает при быстром приложении к кристаллу или снятии с него механического напряжения [1-6], а в ферромагнетиках и ферроэлектриках - включении и выключении, соответственно, магнитного [7-13] или электрического [14] полей. Получаемые при этом упругокалорический, магнитокалорический (МК) и электрокалорический эффекты $\Delta T_{a d}$ достигают 5-20 K [1-14]. Источником адиабатического разогрева или охлаждения сплава служит изотермическое изменение его энтропии при прямом и обратном мартенситных переходах, как фазовых переходов первого рода.

Проведенные в настоящее время исследования (обзоры [7-9]) показали, что магнитокалорические эффекты в ферромагнитных сплавах, таких как $\mathrm{NiMnGa}(\mathrm{Sn}, \mathrm{In})$ с различной степенью нестехиометричности [8,13], наблюдаются в довольно узких температурных интервалах $(5-20 \mathrm{~K})$. Кроме того, они сложным и неоднозначным образом зависят от температуры $T$ и напряженности $H$ магнитного поля при его адиабатических вариациях [8]. На указанные зависимости влияет степень удаленности друг от друга критических температур мартенситного $T_{c}$ и магнитного $T_{C}$ переходов [8] и магнитное состояние мартенсита и аустенита, а также наличие у ферромагнитных кристаллов осей легкого и трудного намагничивания.

Целью настоящей работы состоит в теоретическом анализе магнитокалорического эффекта в ферромагнитных сплавах гейслеровсго типа, испытывающих переход из кубической фазы решетки в тетрагональную ее фазу. Для анализа и моделирования эффекта использована теория размытых термоупругих мартенситных переходов (РТМП) [15,16], основанная на термодинамических и кинетических соотношениях, и чувствительная к структуре кристалла на мезоуровне. Она успешно применялась недавно для анализа упругокалорического эффекта в этих сплавах [5], а ранее для анализа и моделирования эффекта магнитной памяти формы в ферромагнитных сплавах гейслеровского типа $\mathrm{NiMnGa}(\mathrm{Sn}, \mathrm{In}) \quad[17,18]$. В разделе 2 приведены основные термодинамические соотношения теории РТМП, определяющие изотермическое изменение энтропии $\Delta S$ ферромагнитного кристалла и величину магнитокалорического эффекта $\Delta T_{a d}$ при адиабатическом включении и выключении магнитного поля. В третьем разделе эти соотношения используются для анализа магнитокалорического эффекта при ориентации магнитного поля в направлении оси легкого намагничивания [001], в четвертом разделе - при наличии в кристалле направлений легкого и трудного намагничивания. 


\section{2. Магнитокалорический эффект и теория РТМП}

Изотермическое изменение энтропии кристалла $\Delta S$, определяющее величину магнитокалорического эффекта, зависит от температуры кристалла $T$ и приложенного к ферромагнитному кристаллу магнитного поля $H$. Эта зависимость согласно термодинамическому соотношению Максвелла [9]:

$$
\left(\frac{\partial S}{\partial B}\right)_{T}=\left(\frac{\partial M}{\partial T}\right)_{B}
$$

определяется интегралом $[8,9,13]$ :

$$
\Delta S(T, B)=\int_{0}^{B}\left(\frac{\partial M}{\partial T}\right)_{B} d B,
$$

где $M-$ намагниченность кристалла, $B=\mu_{0} H, \mu_{0}-$ магнитная проницаемость. Принимая во внимание термодинамически эквивалентное (1a) соотношение между переменными $S, M, B$ и $T$

$$
\left(\frac{\partial S}{\partial M}\right)_{T}=\left(\frac{\partial B}{\partial T}\right)_{M}
$$

получаем альтернативную (1b) зависимость изотермического изменения энтропии кристалла $\Delta S$ от магнитного поля $B$ и температуры

$$
\Delta S(T, B)=\int_{0}^{M}\left(\frac{\partial B}{\partial T}\right)_{M} d M .
$$

Согласно теории РТМП намагниченность изменяется пропорционально относительной объемной доле $\varphi_{M}$ кристалла, занятой мартенситом. С учетом того, что аустенит также может являться ферромагнетиком с относительной объемной долей $\varphi_{A}=1-\varphi_{M}$, намагниченность кристалла в целом описывается уравнением

$$
\begin{aligned}
M(T, B) & =m_{m} \varphi_{M}(T, B)+m_{a}\left(1-\varphi_{M}(T, B)\right) \\
& =m_{a}+\left(m_{m}-m_{a}\right) \varphi_{M}(T, B),
\end{aligned}
$$

где $m_{m}$ и $m_{a}$ - магнитные моменты, соответственно, мартенсита и аустенита.

При одностадийном характере мартенситного перехода объемная доля мартенсита определяется термодинамическим соотношением $[15,16]$ :

$$
\varphi_{M}=\frac{1}{1+\exp \left(\Delta U / k_{\mathrm{B}} T\right)},
$$

где $\Delta U=\omega \Delta u-$ изменение свободной энергии сплава при образовании в нем зародыша мартенситной фазы объемом $\omega, \Delta u$ - объемная плотность свободной энергия фазового перехода,

$$
\Delta u=q_{0} \frac{T-T_{c 0}}{T_{c 0}}-\Delta m_{M} B
$$

Здесь $q_{0}=\Delta S_{c} T_{c 0}-$ теплота перехода, $T_{c 0}-$ температура мартенситного перехода в отсутствие магнитного поля, $\Delta S_{c}-$ изменение энтропии при мартенситном переходе, $k_{\mathrm{B}}-$ постоянная Больцмана, $\Delta m_{M A} B-$ объемная плотность магнитной энергии мартенсита из-за разницы намагниченностей мартенсита и аустенита $\Delta m_{M A}=\Delta m_{m a} V_{0}$, где $\Delta m_{m a}=m_{m}-m_{a}-$ разница их магнитных моментов, $V_{0}-$ атомный объем сплава. Для дальнейшего удобно записать выражение в экспоненте (3a) в безразмерных переменных

$$
\varphi_{M}(\bar{T}, \bar{B})=\frac{1}{1+\exp [\bar{\omega}(\bar{T}-1-\bar{B})]},
$$

где $\bar{T}=T / T_{c 0}, \bar{B}=B / B_{M}, B_{M}=q_{0} / \Delta m_{M A}, \bar{\omega}=q_{0} \omega / k_{\mathrm{B}} T$ $\approx q_{0} \omega / k_{\mathrm{B}} T_{c 0}$. Решая (5а) относительно поля $B$, получаем его зависимость от температуры и концентрации мартенсита:

$$
B=B_{M}\left(\frac{T}{T_{c 0}}-1+\frac{1}{\bar{\omega}} \ln \left(\frac{\varphi_{M}}{1-\varphi_{M}}\right)\right) .
$$

Частная производная по температуре относительно $B$ равна коэффициенту Клаузиаса-Клапейрона,

$$
\left(\frac{\partial B}{\partial T}\right)_{\varphi_{M}}=\frac{B_{M}}{T_{c 0}}=\frac{q_{0}}{\Delta m_{M A} T_{c 0}} .
$$

В результате, для изотермического изменения энтропии кристалла имеем согласно $(2 \mathrm{~b})$ соотношение

$$
\begin{gathered}
\Delta S_{M}(T, B)=\frac{q_{0}}{\Delta m_{M A} T_{c 0}} \Delta M, \\
\Delta M(T, B)=M(T, B)-M(T, 0) .
\end{gathered}
$$

Далее, принимая во внимание связь магнитокалорического эффекта $\Delta T_{a d}^{M}$ с изотермическим изменением энтропии $\Delta S_{M}$,

$$
\Delta T_{a d}^{M}=\Delta S_{M} T_{c 0} / C_{p},
$$

получаем для него окончательное соотношение

$$
\begin{gathered}
\Delta T_{a d}^{M}(\bar{T}, \bar{B})=\frac{q_{0}}{C_{p}} \Delta \varphi_{M}(\bar{T}, \bar{B}), \\
\Delta \varphi_{M}(\bar{T}, \bar{B})=\varphi_{M}(\bar{T}, \bar{B})-\varphi_{M}(\bar{T}, 0),
\end{gathered}
$$

где $C_{p}=V_{0} C_{p}$ - удельная теплоемкость сплава. Согласно уравнению (7b) магнетокалорический эффект зависит от теплоты мартенситного перехода $q_{0}$ и от количества мартенсита $\Delta \varphi_{M}(T, B)$, создаваемого магнитным полем. Уравнение $(1 \mathrm{~b})$ для изотермической энтропии, учитывающее ее зависимость не только от количества мартенсита, но и от намагниченности кристалла, будет использовано в разделе 4 при обсуждении влияния магнитной анизотропии кристалла на магнитокалорический эффект. 


\section{3. Магнитокалорический эффект в отсутствие магнитной анизотропии}

Сплавы $\mathrm{Ni}_{50+x+y} \mathrm{Mn}_{25-x}(\mathrm{Ga}, \mathrm{Sn}, \mathrm{In})_{25-y}$ с разной степенью отклонения от стехиометрического состава $(x+y=0-6 \%)$ оказывают на магнитные свойства сплава и параметры магнитокалорического эффекта разнообразное и сложное влияние $[8,12,13]$. Высокотемпературная фаза стехиометрического состава сплава $\mathrm{Ni}_{2} \mathrm{MnGa}$ имеет ОЦК решетку, которая при температурах ниже $200 \mathrm{~K}$ превращается в тетрагональный мартенсит, что сопровождается деформацией сжатия кристалла $\varepsilon_{m}=6 \%$ в направлении оси [001]. Отклонение состояния сплава от стехиометрического состава сопровождается ростом температуры мартенситного перехода $T_{c}$ выше комнатной $[19,20]$ вплоть до достижения температурой $T_{c}$ температуры $T_{C}=340-370 \mathrm{~K}$ перехода ферромагнитного состояния указанных сплавов в парамагнитное состояние. Кристаллы сплава анизотропны относительно направления приложения к ним магнитного поля. Приложение поля вдоль кубических осей (010) и (100) требует более высокой напряженности поля по сравнению с направлением [001] для достижения намагниченности кристалла насыщения.

В настоящем разделе, анализ магнетокалорического эффекта произведен в условиях, не осложненных магнитно-анизотропными эффектами и зависимостью магнитных моментов мартенсита $m_{m}$ и аустенита $m_{a}$ от величины магнитного поля. На рис. 1, $a$ кривые демонстрируют в безразмерных координатах зависимость намагниченности кристалла от температуры согласно уравнению (3) вблизи температуры мартенситного перехода $\bar{T}=0.9-1.2$ при трех значениях приведенной индукции $\bar{B}=0,0.5,0.8$ и значении безразмерного элементарного объема зародыша мартенсита $\bar{\omega}=50$,

$$
\bar{M}(\bar{T}, \bar{B})=\bar{m}_{a}+\left(1-\bar{m}_{a}\right) \varphi_{M}(\bar{T}, \bar{B}),
$$

где $\bar{M}=M / m_{m}, \bar{m}_{a}=m_{a} / m_{m}=0.2$. На рис. $1, a$ пунктир $a b$ демонстрирует наклон кривых $M(\bar{T})$ при температуре $\bar{T}_{c}=T_{c} / T_{c 0}$,

$$
\begin{gathered}
\left(\frac{\partial \bar{M}}{\partial \bar{T}}\right)_{\bar{T}_{c}}=-\bar{\omega}\left(1-\bar{m}_{a}\right) \varphi_{c}\left(1-\varphi_{c}\right), \\
\varphi_{c}=\frac{1}{2}\left(\varphi_{m}+\varphi_{a}\right),
\end{gathered}
$$

где $\varphi_{m}=M_{m}^{m} / m_{m}, \varphi_{a}=M_{a}^{a} / m_{a}, M_{m}^{m}$ и $M_{a}^{a}$ - предельные значения намагниченностей мартенсита и аустенита. Температурный интервал (размытость по температуре) мартенситного перехода $\Delta \bar{T}$ определяется соотношением

$$
|\Delta \bar{T}| \approx\left(\bar{M}_{m}-\bar{M}_{a}\right) /(\partial \bar{M} / \partial \bar{T})_{\bar{T}_{c}}=\frac{\varphi_{m}-\varphi_{a}}{\bar{\omega} \varphi_{c}\left(1-\varphi_{c}\right)} .
$$

Согласно (9) температурный интервал перехода тем больше, чем меньше элементарный объем фазового
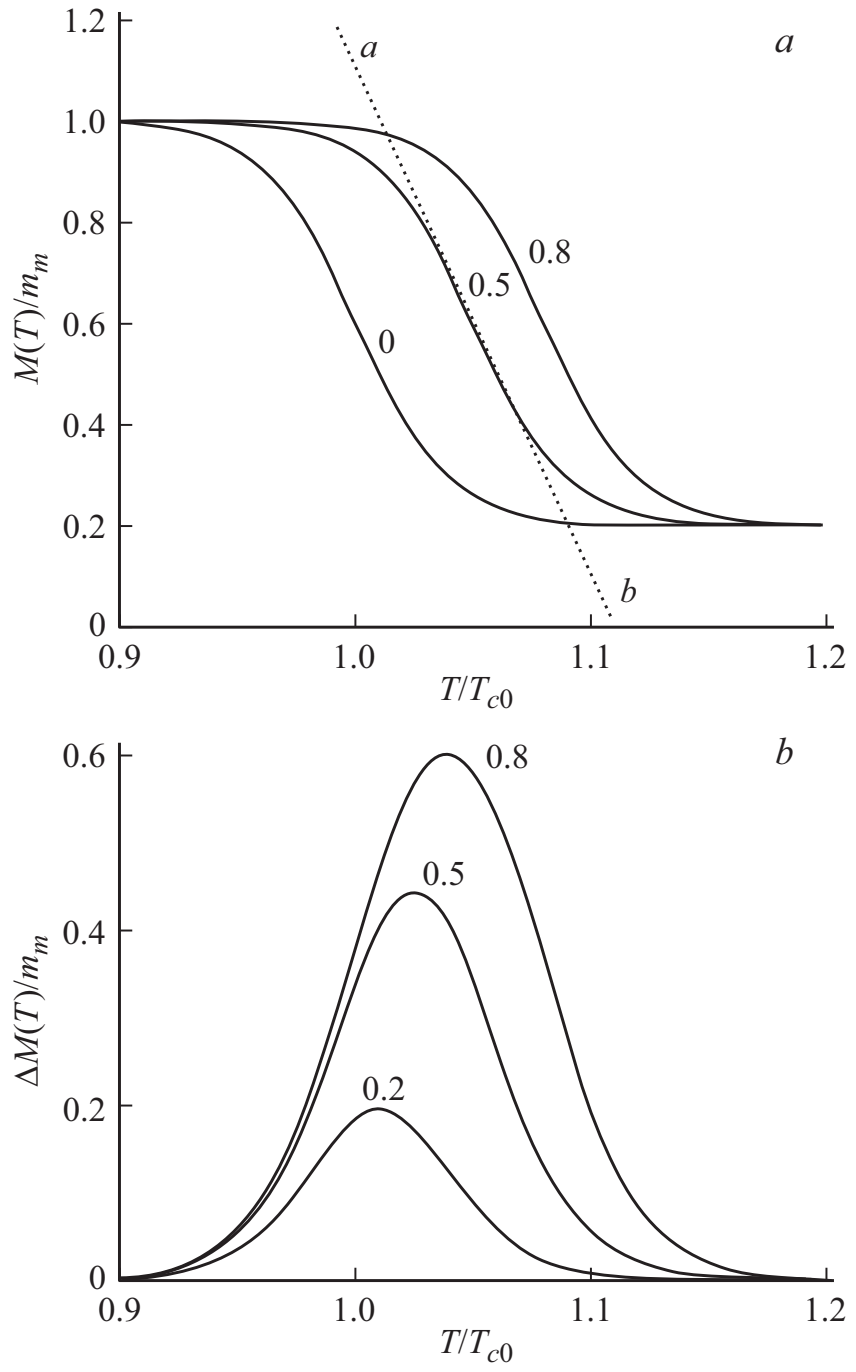

Рис. 1. Температурные зависимости намагниченности $\bar{M}=M / m_{m}(a)$ и разницы намагниченностей $\Delta \bar{M}=\Delta M / m_{m}(b)$ кристалла сплава согласно уравнениям (3) и (6) при трех значениях индукции $\bar{B}=B / B_{M}$ (цифры у кривых) и $\bar{\omega}=50$.

превращения, $\Delta T \sim 1 / \bar{\omega}$. При $\varphi_{a}=0$ и $\varphi_{m}=1$ температурный интервал максимален и равен $(4 / \bar{\omega}) T_{c 0}$.

Магнитокалорический эффект $\Delta T_{a d}^{M} \sim \Delta S_{M}$ и изотермическое изменение энтропии $\Delta S_{M}$ при адиабатическом скачке магнитного поля $B$ зависят согласно уравнениям (6) и (7) от разницы $\Delta M(T, B)$ намагниченности кристалла в магнитном поле и в его отсутствие. Эта разница определяется, в основном, изменением объемной концентрации мартенсита в кристалле $\Delta \varphi_{M}(T, B)$ (7). Рис. $1, b$ демонстрирует зависимость разницы намагниченностей $\Delta \bar{M}=\Delta M / m_{m}$ от температуры $\bar{T}=T / T_{c 0}$ согласно указанным на рис. $1, a$ значениям индукции $\bar{B}$ и элементарного объема превращения $\bar{\omega}$. Разница намагниченностей увеличивается с ростом величины магнитного поля и элементарного объема превращения и достигает максимального значения $\Delta M_{\max }\left(T_{\max }, B\right)$ при температуре $\bar{T}_{\max }=1+\bar{B}$. Зависи- 


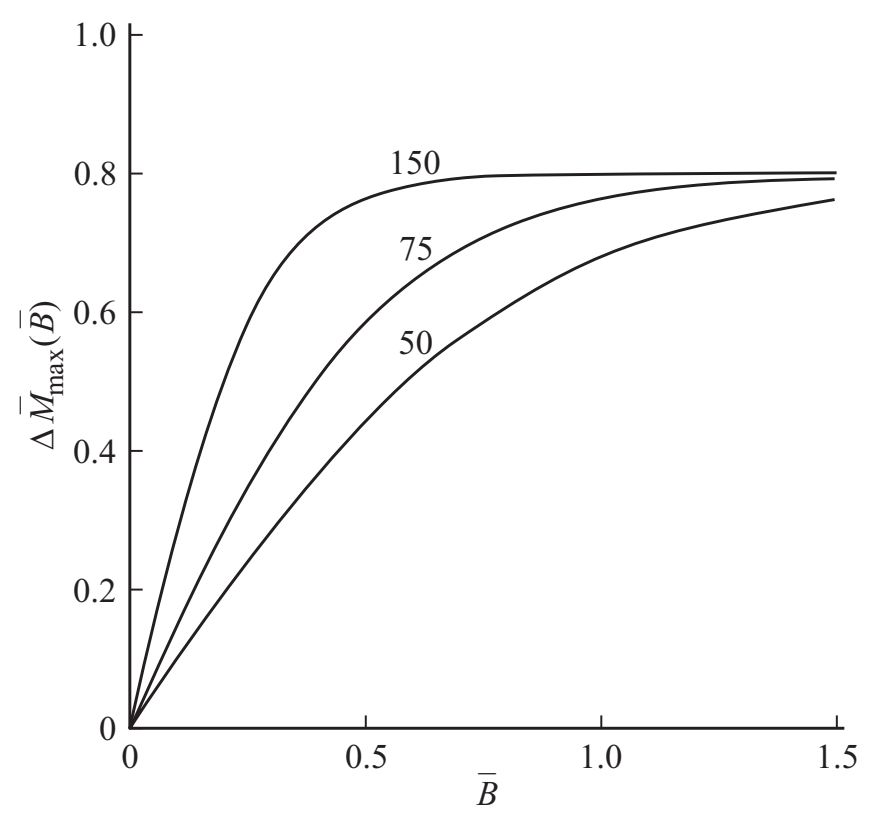

Рис. 2. Зависимость максимального изменения намагниченности кристалла $\Delta \bar{M}_{\max }(\bar{B})$ от магнитного поля $\bar{B}=B / B_{M}$ при трех значениях безразмерного элементарного объема превращения $\bar{\omega}=50,75$ и 150 (цифры у кривых).

мость $\Delta \bar{M}_{\max }(B)$ при трех значениях $\bar{\omega}=50,75$ и 150 приведена на рис. 2. Результаты расчета показывают, что с ростом величины индукции магнето-калорический эффект $\Delta T_{a d}^{M} \sim \Delta S^{M} \sim \Delta M_{\max }(B, \omega)$ достигает насыщения тем быстрее, чем больше величина элементарного объема превращения $\omega$, т.е. чем меньше температурная ширина мартенситного перехода $\Delta T \sim 1 / \omega$. Видно также, что в отличие от эластокалорического эффекта, магнитокалорический эффект наблюдается в довольно узком температурном интервале $\approx 0.1 T_{c 0}=20-30 \mathrm{~K}$ при $T_{c 0}=200-300 \mathrm{~K}$.

\section{4. Влияние магнитной анизотропии на магнитокалорический эффект}

Из экспериментов [8,13] следует, что при ориентации магнитного поля вдоль оси легкого намагничивания кристалла [001] отклонение от стехиометрического состава сплава $\mathrm{Ni}-\mathrm{Mn}-\mathrm{Ga}$ в результате роста концентрации атомов Ni и соответствующей убыли атомов Mn сопровождается ростом критической температуры мартенситного перехода $T_{c}$. Кроме того, изменяется соотношение между намагниченностью мартенсита и аустенита. При концентрации Ni меньше 51.5\% намагниченность мартенсита оказывается меньше, чем аустенита. Но в обоих случаях она нелинейно изменяется с ростом величины магнитного поля $[8,13]$ и зависит от ориентации магнитного поля относительно оси легкого намагничивания кристалла. Двойникование мартенсита с образованием пяти- или семислойных структурных доменов с грани- цами, служащими одновременно границами магнитных доменов, является источником возникновения магнитных напряжений $\sigma_{M}(B)$ и энергии магнитной анизотропии $W_{a}(B)=\varepsilon_{t w} \sigma_{M}(B)[18,22]$, где $\varepsilon_{t w}-$ деформация двойникования при структурной перестройке решетки. В результате, для намагниченности кристалла сплава с ЭПФ имеем соотношение

$$
\begin{aligned}
M(T, B) & =m_{m}(B) \varphi_{M}(T, B)+m_{a}(B)\left(1-\varphi_{M}(T, B)\right) \\
& =m_{a}(B)+\left(m_{m}(B)-m_{a}(B)\right) \varphi_{M}(T, B)
\end{aligned}
$$

Концентрация мартенсита в уравнении (10а) в общем случае может зависеть от энергии магнитной анизотропии $W_{a}$ (Приложение А):

$$
\begin{aligned}
& \varphi_{M}(T, B) \\
& =\frac{1}{1+\exp \left[\bar{\omega}\left(\left(T-T_{c 0}\right) / T_{c 0}-1-\left(\Delta m(B) B+W_{a}(B)\right) / q_{0}\right)\right]},
\end{aligned}
$$

а магнитные моменты мартенсита $m_{m}$ и аустенита $m_{a}$ от магнитного поля [23]. При этом магнитный момент мартенсита зависит еще от ориентации магнитного поля относительно оси легкого намагничивания [001],

$$
\begin{gathered}
m_{m}(B, \beta)=m_{[001]}^{\infty} \operatorname{th}\left(B / B_{[001]}\right) \cos \beta^{2} \\
+m_{[100]}^{\infty} \operatorname{th}\left(B / B_{[100]}\right) \sin \beta^{2}, \\
m_{a}(B)=m_{a}^{\infty} \operatorname{th}\left(B / B_{a}\right) .
\end{gathered}
$$

Здесь $\beta$ - угол между осью [001] кристалла и направлением магнитного поля, $m_{m}^{\infty}$ и $m_{a}^{\infty}-$ магнитные моменты при насыщении кристалла магнитным полем, $B_{[100]}=k_{\mathrm{B}} T / m_{[100]}^{\infty}, B_{[001]}=k_{\mathrm{B}} T / m_{[001]}^{\infty}$, $B_{a}=k_{\mathrm{B}} T / m_{a}^{\infty}[23]$.

Рис. 3, $a$ демонстрирует результаты расчета магнитных моментов мартенсита в направлении осей легкого $([001], \beta=0)$ и трудного $([100], \beta=\pi / 2)$ намагничивания кристалла согласно соотношениям (11). На рис. $3, b$ приведена разница $\Delta m(B)$ магнитных моментов мартенсита $m_{m}(B)$ и аустенита $m_{a}(B)$. Видно, что указанная разница существенно зависит от ориентации магнитного поля относительно направления оси легкого намагничивания кристалла. Приведенные на рис. 3 результаты получены при следующих значениях параметров: $m_{[001]}^{\infty}=m_{[100]}^{\infty}=10, m_{a}^{\infty}=9.5 \mathrm{~A} \cdot \mathrm{m}^{2}, B_{[001]}=1$, $B_{[100]}=2$ и $B_{a}=1$ T (Tesla). Обращает на себя также внимание, что разница магнитных моментов мартенсита и аустенита $\Delta m(B)=m_{m}(B)-m_{a}(B)$ неоднозначно зависит от величины магнитного поля при ориентации его в направлении оси трудного намагничивания кристалла.

Существенное различие между направлениями поля вдоль осей [001] и [100] демонстрируют и приведенные на рис. 4 температурные зависимости намагниченности кристалла согласно уравнениям (10). В первом 
случае (рис. 4,a) намагниченность мартенсита чуть выше намагниченности аустенита, во втором же случае (рис. $4, b$ ) она существенно выше у аустенита. Такой вид температурной зависимостей намагниченности характерен для кристаллов сплава $\mathrm{Ni}_{49.5} \mathrm{Mn}_{25.4} \mathrm{Ga}_{25}[8,13]$, близкого к стехиометрическому составу. Его особенностью является также слабая зависимость критической температуры мартенситного перехода $T_{c}$ от магнитного поля, по крайней мере, при не слишком больших полях. Это обстоятельство учтено при построении кривых на рис. 4, оно означает, что основное изменение намагниченности кристалла при росте величины магнитного поля связано не с изменением объемной доли мартенсита (раздел 3), a с ростом его магнитных моментов.

Адиабатическое включение и выключение магнитного поля приводит к изменению структурного состояния сплава (переходу аустенит в мартенсит и к обратному
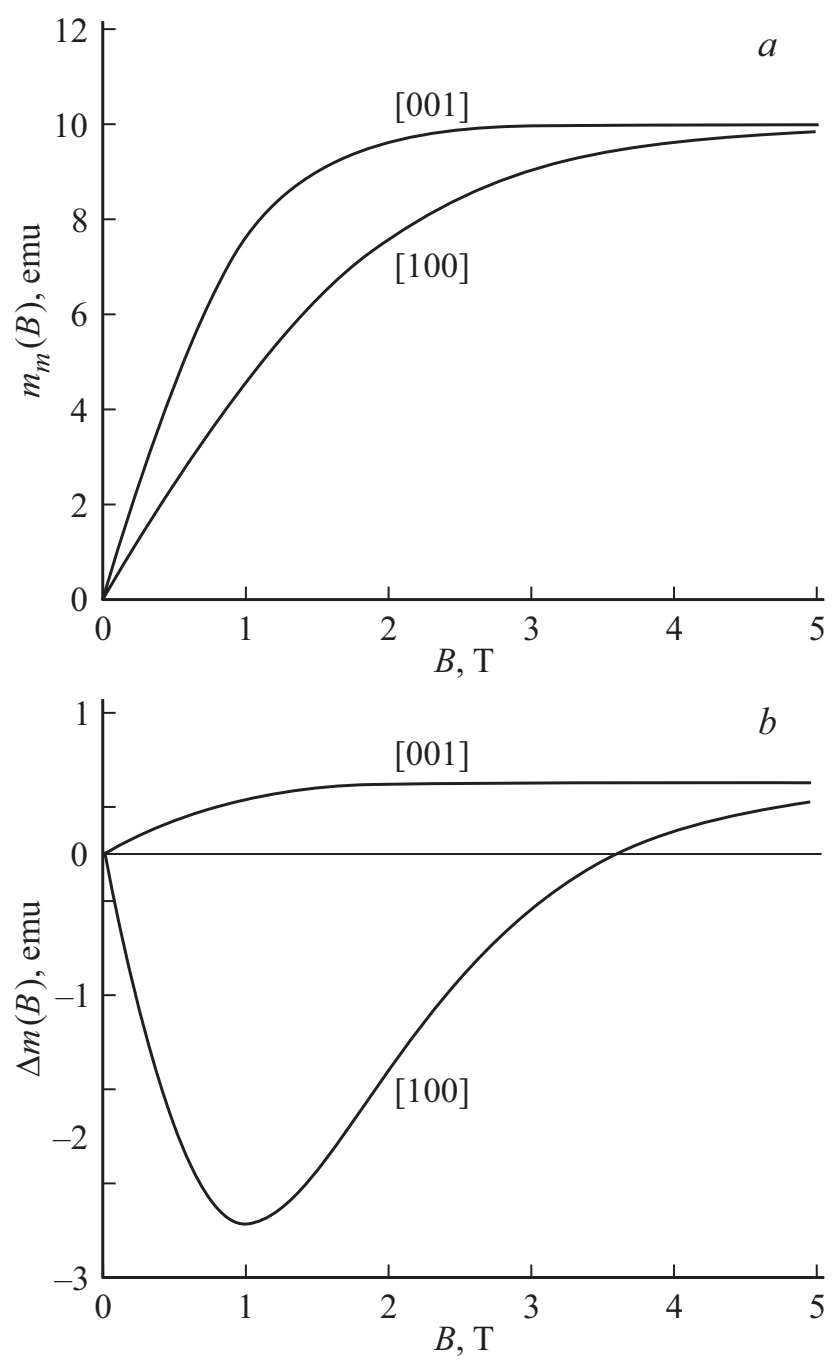

Pис. 3. Зависимость от магнитного поля $B$ магнитных моментов мартенсита $(a)$ и разницы $\Delta m$ магнитных моментов мартенсита и аустенита $(b)$ при ориентации поля вдоль оси, соответственно, легкого [001] и трудного [100] намагничивания кристалла.
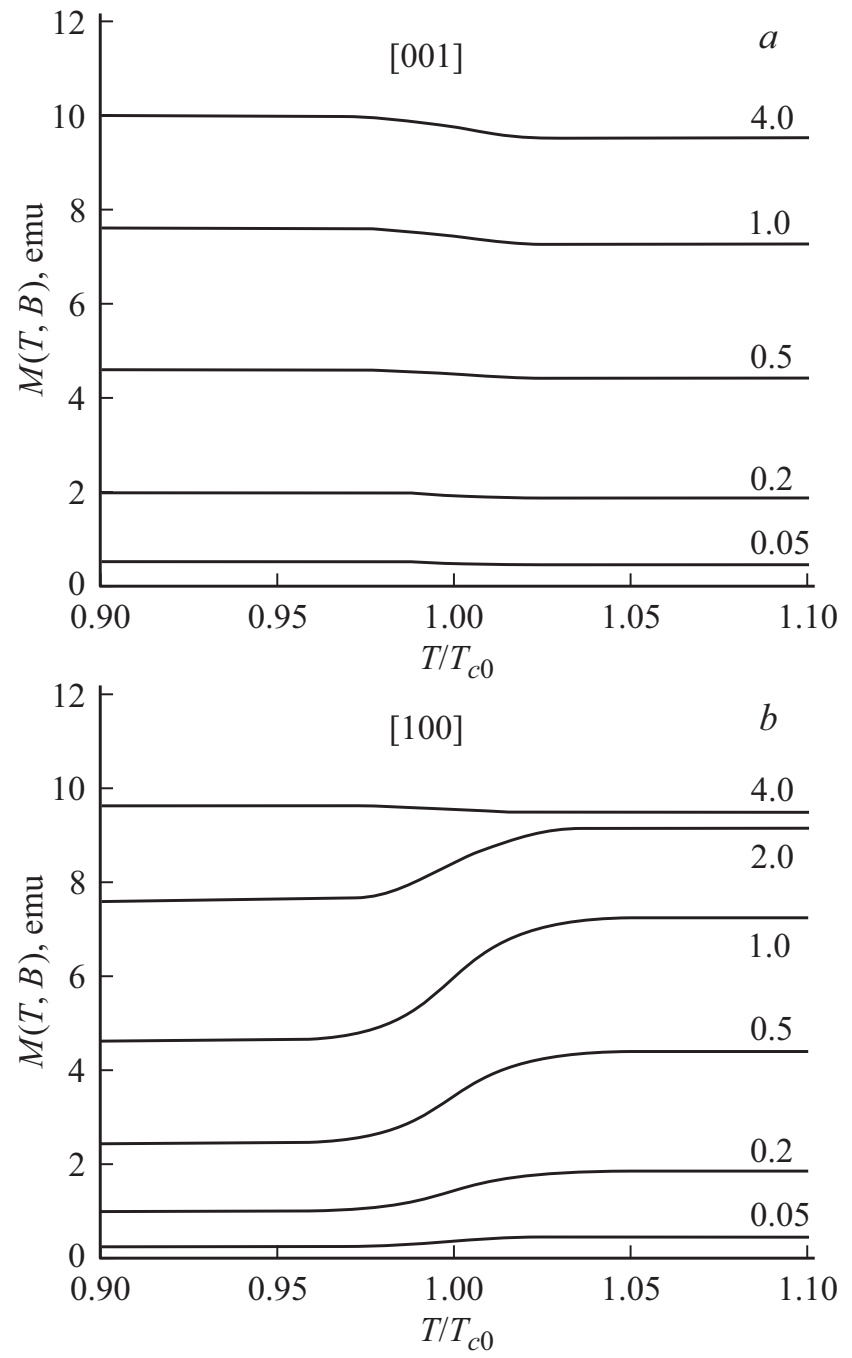

Рис. 4. Температурные зависимости намагниченности кристалла при ориентации магнитного поля вдоль оси, соответственно, легкого [001] (a) и трудного [100] (b) намагничивания кристалла. Цифры у кривых - величина магнитного поля $B$ в единицах $T$.

их переходу) и сопровождается изменением намагниченности кристалла (уравнение (10a)):

$$
\Delta M(T, B)=\left(m_{m}(B)-m_{a}(B)\right) \varphi_{M}(T, B)
$$

Согласно термодинамическому соотношению (1b) изотермическое изменение энтропии кристалла при этом составляет:

$$
\begin{aligned}
\Delta S(T, B, \beta) & =\int_{0}^{B}\left(\frac{\partial \Delta M}{\partial T}\right)_{B} d B \\
& =\left(\frac{\partial \varphi_{M}}{\partial T}\right)_{B} \int_{0}^{B}\left(m_{m}(B, \beta)-m_{a}(B)\right) d B,
\end{aligned}
$$




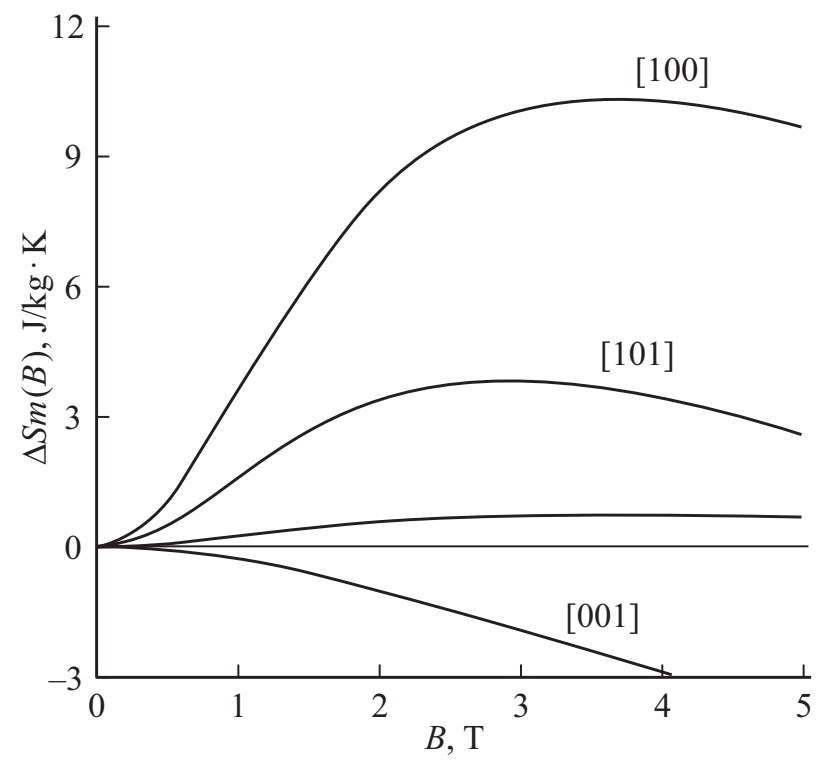

Рис. 5. Зависимость изотермической энтропии $\Delta S$ от магнитного поля $B$ при его адиабатическом включении и указанных на рисунке направлениях магнитного поля в кристалле сплава.

где $[15,16]$

$$
\left(\frac{\partial \varphi_{M}}{\partial T}\right)_{B}=-\bar{\omega} T_{c}^{-1} \varphi_{M}\left(1-\varphi_{M}\right) .
$$

В результате, получаем следующее соотношение для изменения энтропии кристалла при магнитоструктурном переходе

$$
\Delta S(T, B, \beta)=-\bar{\omega} T_{c}^{-1} \varphi_{M}(T)\left(1-\varphi_{M}(T)\right) \Delta M_{m}^{a}(B, \beta) .
$$

Производная $\left(\partial \varphi_{M} / \partial T\right)_{B}$ вынесена за знак интеграла (13a), поскольку предполагается в соответствие с экспериментом $[8,13]$, что магнитное поле не существенно влияет на концентрацию мартенсита, но оказывает существенное влияние на кинетику намагничивания кристалла $\Delta M_{m}^{a}(B, \beta)$ (Приложение В). Согласно уравнению (13b) производная по температуре достигает максимальной величины $\bar{\omega} / 4 T_{c 0}$ при объемной доле мартенсита $\varphi_{M}=1 / 2$, т.е. при критической температуре перехода $T=T_{c 0}$. На рис. 5 показаны результаты расчета согласно уравнениям (13) зависимости от поля $B$ изотермического изменения энтропии кристалла $\Delta S$ при температуре $T=T_{c 0}=200 \mathrm{~K}, \bar{\omega}=100$ и указанных выше параметрах намагничивания мартенсита и аустенита (рис. 3 и 4). Кривые на рис. 5 демонстрируют зависимости $\Delta S(B)$ для трех ориентаций магнитного поля относительно осей кристалла. Видно, что при ориентации поля в направлении оси легкого намагничивания [001] энтропия имеет отрицательный знак и не велика по сравнению с ее положительными значениями при ориентации магнитного поля вдоль осей более трудного намагничивания кристалла. Ее положительное значение на рис. 5 означает, что адиабатическое включение магнитного поля сопровождается нагревом кристалла. Очевидно, что при адиабатическом выключении магнитного поля будет наблюдаться противоположный, рефрижираторный магнитокалорический эффект, $\Delta S<0$, т. е. снижение температуры кристалла, $\Delta T_{a d}<0$. Согласно формуле (7a) магнитокалорический эффект $\Delta T_{a d}$ прямо пропорционален изменению энтропии $\Delta S$ испытывающего мартенситное превращение кристалла. В рассматриваемом в настоящем разделе случае он описывается уравнением

$$
\begin{aligned}
\Delta T_{a d}(T, B, \beta) & =\frac{T_{c 0}}{C_{p}} \Delta S(T, B, \beta) \\
& =-\frac{\bar{\omega}}{C_{p}} \varphi_{M}(T)\left(1-\varphi_{M}(T)\right) \Delta M_{m}^{a}(B, \beta) .
\end{aligned}
$$

На рис. 6 показана его зависимость от температуры $T$ при $T_{c 0}=200 \mathrm{~K}, C_{p}=400 \mathrm{~J} / \mathrm{kg} \cdot \mathrm{K}$ и нескольких значениях магнитной индукции $B$, когда ее рост увеличивает снижение температуры кристалла. Из уравнения (14) следует также, что МК эффект увеличивается с ростом элементарного объема фазового превращения согласно закону $\Delta T_{a d} \sim \bar{\omega} \sim \omega$, т.е. при снижением температурной ширины мартенситного перехода $\Delta T \sim 1 / \omega$. Показанные на рис. 4-6 результаты теоретического анализа магнии токалорического эффекта в рамках теории РТМП хорошо согласуются с результатами его экспериментального исследования в кристаллах сплава $\mathrm{Ni}_{49.5} \mathrm{Mn}_{25.4} \mathrm{Ga}_{25}[8,13]$.

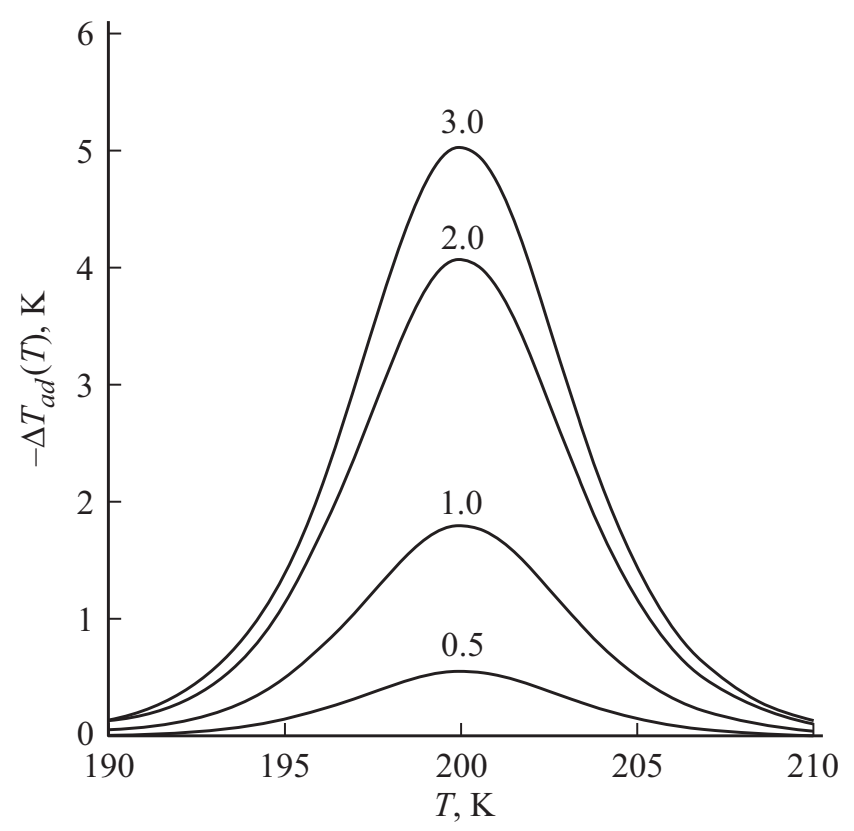

Рис. 6. Температурные зависимости магнитокалорического эффекта $\Delta T_{a d}$ при ориентации магнитного поля вдоль оси трудного намагничивания кристалла [100] и адиабатическом его выключении с указанных на рисунке значений магнитного поля $B$ (цифры у кривых в единицах $T$ ). 

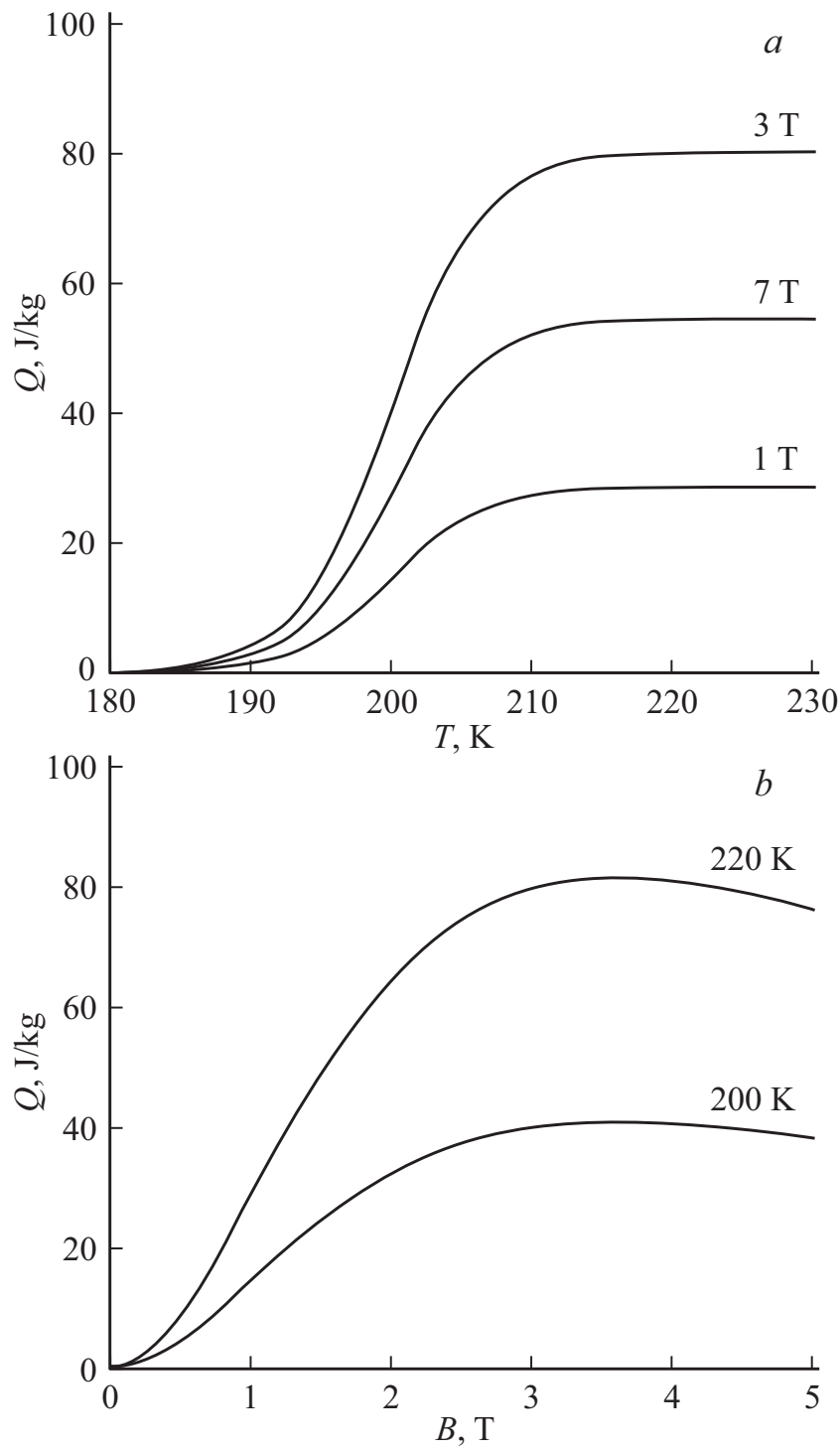

Рис. 7. Температурные $(a)$ и полевые $(b)$ зависимости хладоемкости сплава $Q$ при адиабатическом выключении магнитного поля с указанных на рисунках, соответственно, значений магнитного поля $B$ и температуры $T$ (цифры у кривых).

В заключение раздела получим в рамках теории РТМП важную характеристику магнитокалорического эффекта и твердотельных рефрижераторных устройств, использующих МК эффект. Это количество тепла $Q$, которое устройство способно поглотить, т.е. его хладоемкость,

$$
Q(T, B, \beta)=\int_{0}^{T} \Delta S(T, B, \beta) d T .
$$

Согласно приведенным на рис. 5 результатам расчета, изотермическое изменение энтропии максимально отрицательно при ориентации магнитного поля в направлении оси трудного намагничивания [100]. Ограничиваясь в уравнении (13c) и Приложении В компонентой намагниченности:

$$
\begin{aligned}
\Delta M_{m}^{a}(T, B, \pi / 2)= & m_{[100]}^{\infty} B_{[100]} \ln \left(\operatorname{ch}\left(\frac{B}{B_{[100]}}\right)\right) \\
& -m_{a}^{\infty} B_{a} \ln \left(\operatorname{ch}\left(\frac{B}{B_{a}}\right)\right),
\end{aligned}
$$

получаем после численного интегрирования уравнения (15a) зависимость тепла $Q$ от температуры при трех значениях магнитного поля $B$ (рис. $7, a$ ). На рис. $7, b$ показана соответственно зависимость хладоемкости $Q$ от магнитного поля при двух значениях температуры (цифры у кривых). Видно, что при $B \approx 3.5 \mathrm{~T}$ и $T>220 \mathrm{~K}$ хладоемкость сплава на единицу его массы достигает максимального значения $\approx 80 \mathrm{~J} / \mathrm{kg}$ (атомный вес рассматриваемого (модельного) сплава $\mathrm{Ni}-\mathrm{Mn}-\mathrm{Ga}$ равен $60.5 \mathrm{~g}$ ). Это значение примерно вдвое больше, чем у кристалла $\mathrm{Gd} 43.8 \mathrm{~J} / \mathrm{kg}$ [24].

\section{5. Заключение}

В рамках основанной на термодинамических и кинетических соотношениях теории фазовых переходов первого рода, какими являются структурные мартенситные переходы в сплавах с эффектом памяти формы, теоретически проанализировано влияние магнитного поля на кинетику структурного перехода в кристаллах ферромагнитных сплавов гейслеровского типа, а также связанный с этим влиянием магнитокалорический эффект. Анализ показал, что теория размытых термоупругих мартенситных переходов адекватно описывает, наблюдаемые в эксперименте с кристаллами сплава $\mathrm{Ni}_{49.5} \mathrm{Mn}_{25.4} \mathrm{Ga}_{25}$ [8,13] зависимости магнитокалорического эффекта от температуры и магнитного поля при адиабатических его вариациях.

\section{Приложение A}

Вводя обозначения $f=\cos \beta^{2}$ и $1-f=\sin \beta^{2}$ и используя уравнение $(10 \mathrm{c})$ основного текста и соотношение для магнитного напряжения [22]

$$
\sigma_{\text {magn }}(B)=\varepsilon_{t w}^{-1} \frac{\partial}{\partial f} \int_{0}^{B} m_{m}(B, f) d B,
$$

получаем, что энергия магнитной анизотропии $W_{a}=\varepsilon_{t w} \sigma_{\text {magn }}$ определяется формулой

$$
\begin{aligned}
W_{a}(B)= & m_{[001]}^{\infty} B_{[001]} \ln \left(\operatorname{ch}\left(\frac{B}{B_{[001]}}\right)\right) \\
& -m_{[100]}^{\infty} B_{[100]} \ln \left(\operatorname{ch}\left(\frac{B}{B_{[100]}}\right)\right),
\end{aligned}
$$


При $B \rightarrow \infty$ она линейно зависит от магнитного поля,

$$
\begin{aligned}
W_{a}(B \rightarrow \infty)= & \left(m_{[001]}^{\infty}-m_{[100]}^{\infty}\right) B \\
& +\left(m_{[100]}^{\infty} B_{[100]}-m_{[001]}^{\infty} B_{[001]}\right) \ln 2,
\end{aligned}
$$

а при $m_{[001]}^{\infty}=m_{[100]}^{\infty}=m_{s}$ стремится к предельной величине $[18]$

$$
W_{a}^{\infty}=m_{s}\left(B_{[100]}-B_{[001]}\right) \ln 2 .
$$

\section{Приложение В}

Подставляя в интеграл (13a) основного текста соотношения (10c) и (10d), получаем

$$
\begin{aligned}
\Delta M_{m}^{a}(B, \beta)= & \int_{0}^{B}\left(m_{m}(B, \beta)-m_{a}(B)\right) d B \\
= & m_{[001]}^{\infty} B_{[001]} \ln \left(\operatorname{ch}\left(\frac{B}{B_{[001]}}\right)\right)(\cos \beta)^{2} \\
& +m_{[100]}^{\infty} B_{[100]} \ln \left(\operatorname{ch}\left(\frac{B}{B_{[100]}}\right)\right)(\sin \beta)^{2} \\
& -m_{a}^{\infty} B_{a} \ln \left(\operatorname{ch}\left(\frac{B}{B_{a}}\right)\right)
\end{aligned}
$$

\section{Конфликт интересов}

Автор заявляет об отсутствии конфликта интересов.

\section{Список литературы}

[1] E. Bonnot, R. Romero, L. Mañosa, E. Vives, A. Planes. Phys. Rev. Lett. 100, 125901 (2008).

[2] L. Mañosa, S. Jarque-Farnos, E. Vives, A. Planes. Appl. Phys. Lett. 103, 211904 (2013).

[3] F. Xiao, M. Jin, J. Liu, X. Jin. Acta Mater. 96, 292 (2015).

[4] D. Zhao, F. Xiao, Zh. Nie, D. Cong, W. Sun, J. Liu. Scripta Mater. 149, 6 (2018).

[5] Г.А. Малыгин. ФТТ 64, 255 (2022).

[6] X-M. Huang, L-D. Wang, H-X. Liu, H-L. Yan, N. Jia, B. Yang, Z-B. Li, Yu-D. Zhang, C. Esling, X. Zhao, L. Zuo. Intermetallics 113, 106579 (2019).

[7] K.A. Gschneidner, V.K. Pecharsky, A.O. Tsokol. Rep. Prog. Phys. 68, 1479 (2005).

[8] A. Planes, L. Mañosa, M. Aset. J. Phys.: Condens. Matter 21, 233201 (2009).

[9] T. Brück, J. Phys. D 38, R381 (2005).

[10] L. Mañosa, S. Jarque-Farnos, E. Vives, A. Planes. Appl. Phys. Lett. 103, 211904 (2013).

[11] F. Xiao, M. Jin, J. Liu, X. Jin. Acta Mater. 96, 292 (2015).

[12] F. Hu, B. Shen, J. Sun, G. Wu. Phys. Rev. B 64, 132412 (2001).

[13] J. Marcos, A. Planes, L. Mañosa, F. Casanova, X. Battle, A. Labarta, B. Martinez. Phys. Rev. B 66, 224413 (2002).

[14] A.S. Mishenko, Q. Zhang, J.F. Scott, R.W. Wathmore, N.D. Mathur. Science 311, issue 5765, 1270 (2006).

[15] Г.А. Малыгин. ФТТ 36, 1489 (1994).
[16] Г.А. Малыгин. УФН 171, 187 (2001).

[17] Г.А. Малыгин. ЖТФ 77, 8, 136 (2007).

[18] Г.А. Малыгин. ФТТ 51, 1599 (2009).

[19] А.Н. Васильев, В.Д Бучельников, Т. Такаги, В.В. Ховайло, Э.И. Эстрин. УФН 173, 577 (2003).

[20] M. Richard, J. Feuchtwanger, D. Schlagel, T. Lograsso, S.M. Allen, R.C. O’Handley. Scripta Mater. 54, 1797 (2006).

[21] Г.А. Малыгин. ФТТ 63, 272 (2021).

[22] A.A. Lichachev, A. Sozinov, K. Ullakko. Mater. Sci. Eng. A 378, 513 (2004).

[23] Дж. Смарт. Эффективное поле в теории магнетизма. Мир, M. (1968). $271 \mathrm{c.}$

[24] В.И. Митюк, Н.Ю. Панкратов, Г.А. Говор, С.А. Никитин, А.И. Смаржевская. ФТТ 54, 1865 (2012).

Редактор К.В. Емцев

5 Физика твердого тела, 2022, том 64, вып. 5 